\title{
Dynamic neointimal pattern after drug-eluting stent implantation defined by optical coherence tomography
} Erika Yamamoto ${ }^{a}$, Lei Xing ${ }^{a}$, Takumi Higuma ${ }^{a}$, Yoshiyasu Minami ${ }^{a}$, Krzysztof Bryniarski ${ }^{\mathrm{a}}$, Thomas Zanchin ${ }^{\mathrm{a}}$, Zhao Wang ${ }^{\mathrm{c}}$, Aaron D. Aguirre ${ }^{\mathrm{a}}$, Hang Lee ${ }^{b}$ and Ik-Kyung Jang ${ }^{a, d}$

\begin{abstract}
Objectives Certain neointimal patterns including neoatherosclerosis (NA) are known to be associated with poor clinical outcome. The prevalence and time course of different neointimal patterns have not been studied systematically. The aim of this study was to investigate the serial changes in neointimal pattern after drug-eluting stent implantation.
\end{abstract}

Patients and methods A total of 132 patients with 207 drug-eluting stents, who underwent two follow-up optical coherence tomography studies at 6 and 12 months, were included. Neointimal patterns were categorized as homogeneous, heterogeneous, layered, or NA using optical coherence tomography. Quantitative and qualitative analyses of neointima were carried out.

Results Both at 6 and at 12 months, the homogenous neointima was the predominant type ( $>75 \%)$, followed by the layered and the heterogeneous pattern. At 12 months, progression to NA was observed in $0.6 \%$ of the patients in the homogeneous group, in $5.6 \%$ of the patients in the heterogeneous group, and in $3.9 \%$ of the patients in the layered group. Regression to the homogeneous pattern was

\section{Introduction}

In-stent neoatherosclerosis (NA) is recognized as one of the major contributing factors toward late stent failure that also include very late stent thrombosis and in-stent restenosis [1-3]. Recently, Taniwaki et al. [4] reported that NA was found in $27.6 \%$ of very late stent thrombosis cases. The incidence of NA in second-generation drug-eluting stent (DES) has been reported to be comparable to that in firstgeneration DES [5,6]. Moreover, NA was also observed even after implantation of a bioresorbable scaffold [7,8]. Therefore, NA is still one of the major concerns after stent implantation. Several studies previously reported predictors of NA and the serial changes in neointima leading to NA [2,6,9-12]. However, only a few studies have described the serial change of neointima to a neointima pattern other than NA. The aim of our study was to investigate the detailed serial changes in neointimal patterns.

\section{Patients and methods}

\section{Study population}

Study participants were retrospectively selected from the Massachusetts General Hospital (MGH) optical observed in $5.6 \%$ of the patients in the heterogeneous group and $11.5 \%$ of the patients in the layered group.

Conclusion The homogenous neointima is the predominant pattern both at 6 and at 12 months. The neointimal pattern changed between 6 and 12 months in $10.6 \%$ of stents. Further studies are needed to understand the mechanisms of these neointimal changes and their clinical significance. Coron Artery Dis 28:557-563 Copyright (C) 2017 Wolters Kluwer Health, Inc. All rights reserved.

Coronary Artery Disease 2017, 28:557-563

Keywords: neoatherosclerosis, neointima, optical coherence tomography

${ }^{a}$ Cardiology Division, ${ }^{b}$ Biostatistics Center, Massachusetts General Hospital, Harvard Medical School, Boston, 'Department of Electrical Engineering and Computer Science and Research Laboratory of Electronics, Massachusetts Institute of Technology, Cambridge, Massachusetts, USA and Division of Cardiology, Kyung Hee University Hospital, Seoul, Republic of Korea

Correspondence to Ik-Kyung Jang, MD, PhD, Cardiology Division, Massachusetts General Hospital, Harvard Medical School, GRB 800, 55 Fruit Street, Boston, 02114 MA, USA

Tel: +1 617726 9226; fax: +1 617726 7416; e-mail: ijang@mgh.harvard.edu

Received 10 June 2017 Revised 14 June 2017 Accepted 18 June 2017

coherence tomography (OCT) registry, which is an international multicenter registry of patients who underwent OCT of the coronary arteries and involves 20 sites across six countries (http://www.clinicaltrials. gov:NCT01110538). The registry was approved by the institutional review board at each participating site. Written informed consent was obtained from all patients before enrollment. For this study, we identify 137 patients treated with 213 DES, who had two follow-up OCT imaging procedures at 6 months ( \pm 3 months) and 12 months ( \pm 3 months). They also had to have mean neointimal thickness more than $100 \mu \mathrm{m}$ on at least three consecutive cross-sectional images at a $1 \mathrm{~mm}$ interval at the 6-month follow-up. Among these, five patients (six stents) who underwent target lesion revascularization at the time of the 6-month follow-up were excluded (heterogeneous: two stents, layered: four stents). Finally, 207 DEStreated lesions from 132 patients were included in the analysis. The reasons for the 12-month follow-up were recurrent ischemia $(N=22)$ or scheduled follow-up $(N=110)$. 


\section{Optical coherence tomography image acquisition and analysis}

A frequency-domain OCT system (FD-OCT; C7-XR OCT Intravascular Imaging System and ILUMIEN OCT Intravascular Imaging Systems, St. Jude Medical, St. Paul, Minnesota, USA) and a time-domain OCT system (TD-OCT; M2/M3 Cardiology Imaging System, LightLab Imaging Inc., Westford, Massachusetts, USA) were used in this study. The technique of intracoronary OCT imaging has been described previously [13]. All images were digitally stored, deidentified, relabeled, and submitted to MGH (Boston, Massachusetts, USA) for analysis.

For serial comparisons, stents on the 6-month follow-up were matched to those on the 12-month follow-up using longitudinal landmarks such as side branches, calcium, and stent edges. Cross-sectional OCT images were analyzed for qualitative and quantitative parameters. Lumen and stent cross-sectional area (CSA) were measured at the site of maximum neointimal hyperplasia and neointimal CSA was calculated as the stent CSA minus the luminal CSA. Neointimal CSA stenosis rate was calculated as (neointimal CSA/stent CSA) $\times 100 \%$. The neointimal patterns were categorized as (i) homogeneous, a uniform signal-rich band without focal variation or attenuation (Fig. 1a); (ii) heterogeneous, nonuniform optical properties and various backscattering patterns (Fig. 1b); (iii) layered, layers with different optical properties, namely a luminal high scattering layer and adluminal low scattering layer (Fig. 1c); and (iv) NA, lipid-laden neointima (marked signal attenuation with a diffuse border (Fig. 1d) [14-18]. Peri-strut low-intensity area (PLIA) was defined as a region around the struts with lower homogeneous intensity than the surrounding tissue on OCT images without signal attenuation [19,20]. Macrophage infiltration was defined as increased signal intensity accompanied by heterogeneous back shadows as reported previously [21,22]. Neovascularization was defined as small vesicular or tubular structures with a diameter of $50-300 \mu \mathrm{m} \quad[15,16]$. The thrombus was defined as a mass protruding into the lumen and dimension greater than $250 \mu \mathrm{m}$ [16]. For serial comparison, a quantitative assessment was performed using the OC'T off-line analysis software (St. Jude Medical). OCT images were analyzed using off-line analysis software at the MGH OCT Core Laboratory. All OCT images were analyzed by two independent investigators who were blinded to patient information. When there was discordance between the readers, a consensus reading was obtained from a third independent investigator.

\section{Statistical methods}

Categorical variables were reported as $n(\%)$ and compared using the $\chi^{2}$-test or Fisher's exact test. Continuous variables were expressed as mean $\pm \mathrm{SD}$ or median and interquartile range. Quantitative parameters were compared using the paired $t$-test. Qualitative parameters were compared using the McNemar test for the comparison between the 6-month and the 12-month followups. Statistical significance was defined as $P$ value less than 0.05. Statistical analyses were carried out using SPSS 18.0 (SPSS Inc., Chicago, Illinois, USA).

\section{Results \\ Patient characteristics}

At the 6-month follow-up, $162(78.3 \%)$ stents were categorized as homogeneous and $18(8.7 \%)$ stents were categorized as heterogeneous, 26 (12.6\%) stents were categorized as layered, and one $(0.5 \%)$ stent was categorized as NA. Clinical characteristics of the study population are shown in Table 1 . The median time interval from stent implantation to the 6-month follow-up was 191 (142-203) days and that to the 12-month follow-up was 376 (362-385) days.

\section{Serial change of neointimal pattern}

At the 12-month follow-up, $153(73.9 \%)$ stents were categorized as a homogeneous group, $14(6.8 \%)$ stents as heterogeneous, $36(17.4 \%)$ stents as layered, and four $(1.9 \%)$ stents as NA. There was a trend toward a decrease in the homogeneous (78.3-73.9\%) and heterogeneous pattern $(8.7-6.8 \%)$ at the 12-month follow-up, whereas the layered (12.6-17.4\%) and NA (0.5-1.9\%) patterns trended upward (Fig. 2). The changes in the neointimal pattern are shown in Fig. 3. Progression to a nonhomogeneous pattern was observed in $8.0 \%$ of patients with a homogeneous pattern. Progression to NA was observed in $0.6 \%$ of patients with a homogeneous pattern and $4.5 \%$ of patients with nonhomogeneous patterns $(5.6 \%$ of heterogeneous and $3.9 \%$ of patients with a layered pattern $)(P=0.05)$. Also, progression to a layered pattern was observed in $6.8 \%$ of patients with a homogeneous pattern and $16.7 \%$ of patients with a heterogeneous pattern. However, progression to the heterogeneous pattern from the layered pattern was not detected, and was observed only in $0.6 \%$ of patients with a homogeneous pattern. Regression to a homogeneous pattern was observed in $9.1 \%$ of patients with nonhomogeneous neointima $(5.6 \%$ of patients with a heterogeneous pattern and $11.5 \%$ of patients with a layered pattern). The one stent with NA at the 6-month follow-up remained unchanged at the 12-month follow-up. OCT parameters at the 6-month and the 12-month follow-up time points are shown in Table 2. Neointima CSA stenosis was significantly greater and lumen area was smaller at the 12-month follow-up. The prevalence of PLIA decreased significantly from the first to the 12-month follow-up. The representative images are shown in Fig. 4.

\section{Discussion}

The main findings of this study are as follows: (i) homogenous neointima is the predominant pattern both at 6 and at 12 months, (ii) progression to nonhomogeneous neointima was observed in $8.0 \%$ of 
(a)

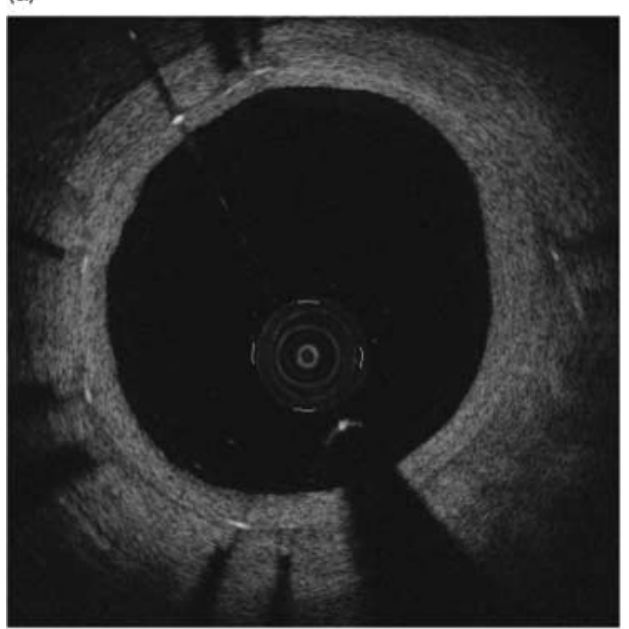

(c)

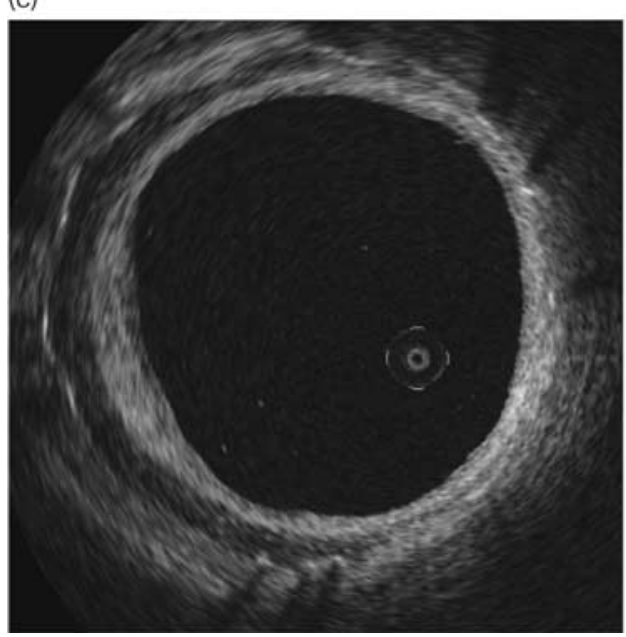

(b)

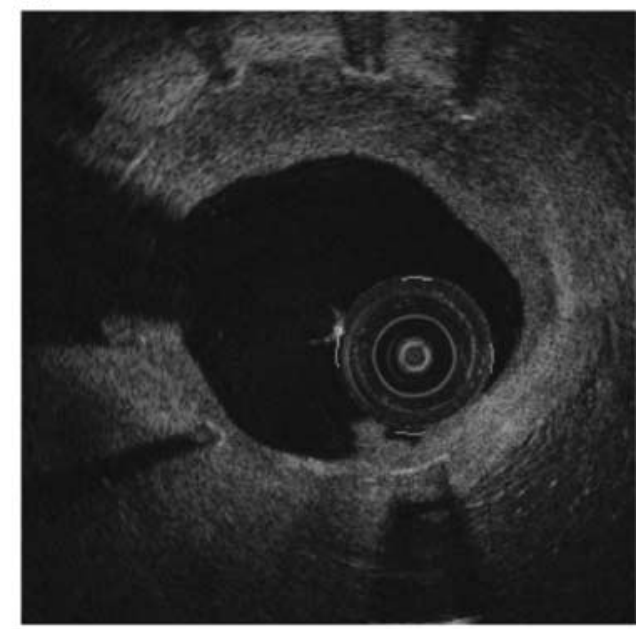

(d)

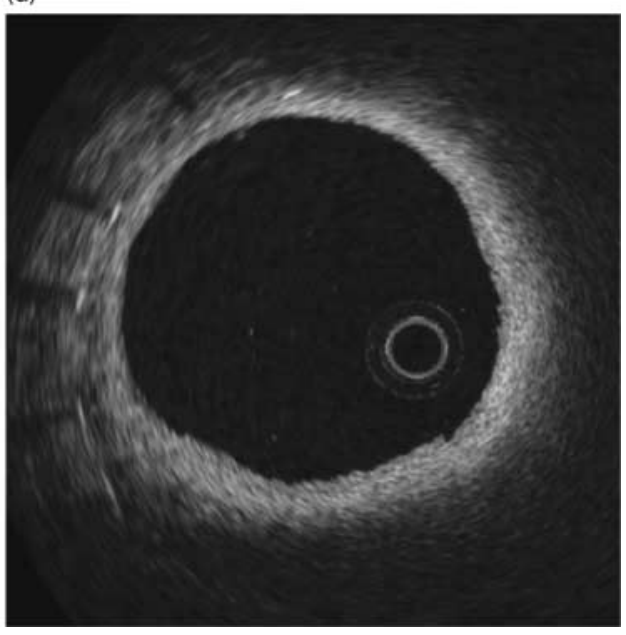

Representative optical coherence tomography images of different neointimal patterns. (a) Homogeneous pattern, a uniform signal-rich band without focal variation or attenuation; (b) Heterogeneous pattern, nonuniform optical properties and various backscattering patterns; (c) Layered pattern, layers with different optical properties, namely, a luminal high scattering layer and adluminal low scattering layer with a visible stent strut;

(d) neoatherosclerosis (NA), lipid-laden neointima with an invisible stent strut.

patients with homogenous neointima at 6 months, (iii) progression to NA was observed in $0.6 \%$ of patients with a homogeneous pattern and $4.5 \%$ of patients with nonhomogeneous neointima at 6 months, and (iv) regression to homogeneous neointima was observed in $9.1 \%$ of patients with nonhomogeneous neointima at 6 months.

\section{Serial changes of the neointimal pattern}

Previous studies reported that the proportion of homogenous neointima decreased and nonhomogenous neointima including NA increased over time [12,23]. Kim et al. [12] reported that $30.3 \%$ of patients with homogeneous neointima developed a nonhomogeneous pattern from 9 months to 2 years. However, they categorized neointima into homogeneous, heterogeneous (this group included classical 'heterogeneous' and 'layered' patterns), and lipid-laden patterns. Therefore, the difference between heterogeneous and layered patterns remains unknown. Lee and colleagues also reported changes in the neointimal pattern and degree of neointimal hyperplasia. In lesions with regression of neointima hyperplasia, conversion to a homogeneous pattern was observed in seven of eight patients with heterogeneous neointima and progression to the layered pattern or NA was not observed. In contrast, progression to layered or NA intima was observed in lesions with progression of neointima [11]. However, the number of cases, especially patients with nonhomogenous neointima, in this study was quite small. Therefore, the serial changes in neointima, in particular the changes in nonhomogeneous neointima, are still unclear. Our data showed that homogeneous and heterogeneous patterns decreased and layered 
Table 1 Patient characteristics at the 6-month follow-up

\begin{tabular}{lc}
\hline Clinical variables & \\
Age (years) & $58.2 \pm 9.8$ \\
Male & $100(75.8)$ \\
BMI (kg/m $\left.{ }^{2}\right)$ & $24.8 \pm 2.7$ \\
Hypertension & $82(62.6)$ \\
Diabetes mellitus & $55(42.0)$ \\
Dyslipidemia & $107(81.1)$ \\
Current smoker & $34(25.8)$ \\
Previous Ml & $42(31.1)$ \\
Presentation & \\
AMI & $8(6.1)$ \\
Unstable angina & $47(35.6)$ \\
Stable angina & $77(58.3)$ \\
Laboratory data & \\
Total cholesterol (mg/dl) & $150.6 \pm 53.1$ \\
LDL-cholesterol (mg/dl) & $83.0 \pm 34.8$ \\
HDL-cholesterol (mg/dl) & $43.4 \pm 15.0$ \\
Triglyceride (mg/dl) & $132.7 \pm 109.6$ \\
Creatinine (mg/dl) & $0.89 \pm 0.27$ \\
HbA1c (\%) & $6.5 \pm 1.4$ \\
Medication at discharge & \\
ACEl/ARB & $49(37.1)$ \\
Statin & $132(100)$ \\
Angiographic variables & 207 stents \\
Location & \\
LAD & $104(50.0)$ \\
LCX & $42(20.3)$ \\
RCA & $61(29.5)$ \\
Stent type & \\
First-generation DES & $181(87.4)$ \\
Second-generation DES & $26(12.6)$ \\
\hline &
\end{tabular}

Values are mean $\pm \mathrm{SD}, n(\%)$, or median (IQR).

$\mathrm{ACEl}$, angiotensin-converting enzyme inhibitor; AMI, acute myocardial infarction; $\mathrm{ARB}$, angiotensin II receptor blocker; DES, drug-eluting stent; HDL, high-density lipoprotein; LAD, left anterior descending artery; LCX, left circumflex artery; LDL, low-density lipoprotein; RCA, right coronary artery.

Fig. 2

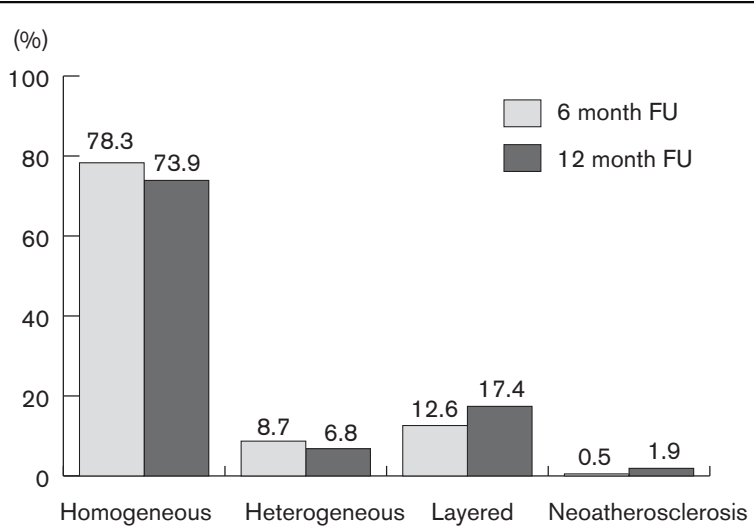

The prevalence of each neointima at the 6-month and the 12-month follow-ups. There was a trend toward a decrease in the prevalence of homogeneous and heterogeneous patterns at the 12-month follow-up (FU) and an increase in the prevalence of layered and neoatherosclerosis patterns.

and NA patterns increased between 6 months and 1 year. Specifically, $6.8 \%$ of patients with a homogeneous pattern and $16.7 \%$ of patients with heterogeneous patterns at 6 months developed a layered pattern at 12 months, and $0.6 \%$ of patients with a homogeneous pattern, $5.6 \%$ of patients with a heterogeneous pattern, and $3.9 \%$ of patients
Fig. 3

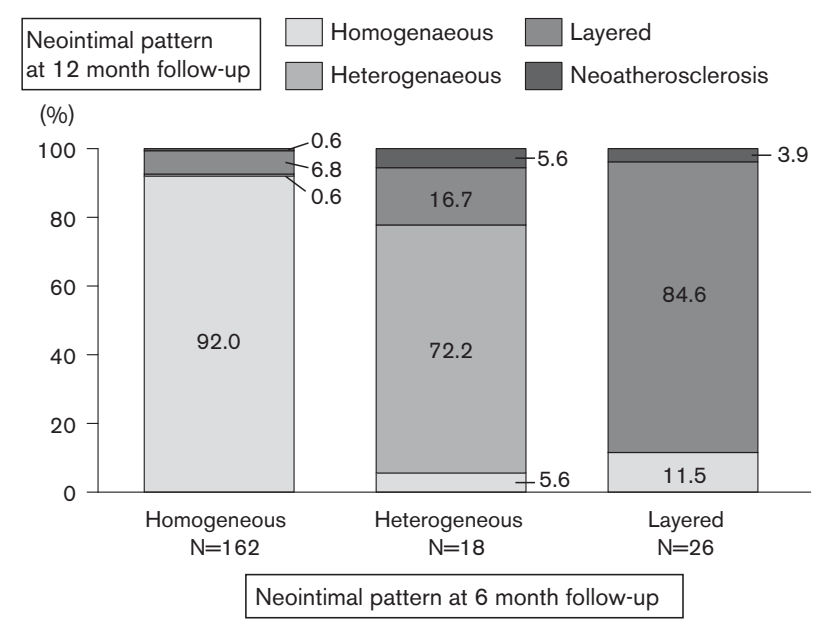

The changes in neointimal patterns between the 6-month and the 12-month follow-ups. Homogeneous patterns at the 6-month follow-up converted into heterogeneous $(0.6 \%)$, layered $(6.8 \%)$, and neoatherosclerosis (NA) $(0.6 \%)$ at the 12-month follow-up. Heterogeneous patterns at the 6-month follow-up converted into homogeneous (5.6\%), layered patterns (16.7\%), and NA $(5.6 \%)$ at the 12 -month follow-up. Layered patterns at the 6 -month follow-up converted into homogeneous $(11.5 \%)$ and NA (3.9\%) patterns at the 12-month follow-up.

Table 2 Quantitative and qualitative optical coherence tomography parameters (6-month and 12-month follow-ups)

\begin{tabular}{|c|c|c|c|}
\hline & 6-Month follow-up & $\begin{array}{l}\text { 12-Month } \\
\text { follow-up }\end{array}$ & $P$ \\
\hline \multicolumn{4}{|l|}{ Quantitative } \\
\hline Lumen area $\left(\mathrm{mm}^{2}\right)$ & $5.5 \pm 2.5$ & $5.1 \pm 2.4$ & $<0.001$ \\
\hline Stent area $\left(\mathrm{mm}^{2}\right)$ & $7.3 \pm 2.5$ & $7.3 \pm 2.6$ & 0.21 \\
\hline Neointimal area $\left(\mathrm{mm}^{2}\right)$ & $1.8 \pm 1.4$ & $2.1 \pm 1.4$ & $<0.001$ \\
\hline Min NIT $(\mu \mathrm{m})$ & $104.8 \pm 117.3$ & $119.4 \pm 117.2$ & 0.06 \\
\hline Mean NIT $(\mu \mathrm{m})$ & $232.8 \pm 179.0$ & $265.1 \pm 183.5$ & $<0.001$ \\
\hline Max NIT ( $\mu \mathrm{m})$ & $364.2 \pm 256.5$ & $419.4 \pm 280.4$ & $<0.001$ \\
\hline $\begin{array}{c}\text { Neointimal CSA } \\
\text { stenosis (\%) }\end{array}$ & $25.6 \pm 18.6$ & $30.6 \pm 18.6$ & $<0.001$ \\
\hline \multicolumn{4}{|l|}{ Qualitative } \\
\hline Neovascularization & $45(21.7)$ & $52(25.1)$ & 0.19 \\
\hline $\begin{array}{l}\text { Peri-strut low-intensity } \\
\text { area }\end{array}$ & $97(46.9)$ & $36(17.4)$ & $<0.001$ \\
\hline Macrophage & $1(0.5)$ & $3(1.4)$ & 0.16 \\
\hline Thrombus & $10(4.8)$ & $13(6.3)$ & 0.41 \\
\hline
\end{tabular}

Values are mean \pm SD or $n(\%)$.

CSA, cross-sectional area; NIT, neointimal thickness.

with layered patterns at 6 months developed NA. The nature of OCT dark appearance (low intensity) areas observed in layered or heterogeneous neointima has been studied. Nakano et al. [24] reported that excessive inflammation, fibrin accumulation, and organized thrombus showed a similar dark appearance in OCT. Shibuya et al. [25] also reported that low-intensity areas with diffuse borders and visible strut in OCT were a proteoglycan-rich myxomatous matrix. Mixtures of these tissues and fibrous tissue create a heterogeneous or a layered pattern. Accurate differentiation of these components on OCT is not possible. Recently, a pathology study reported that both heterogeneous and 


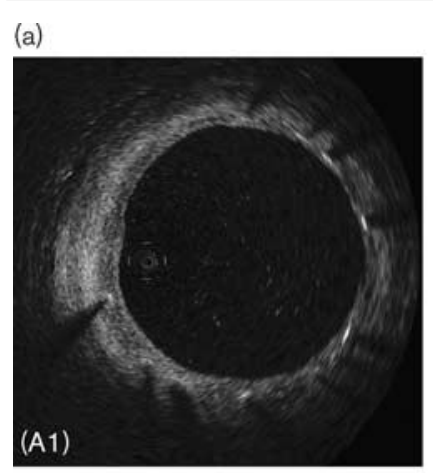

(b)
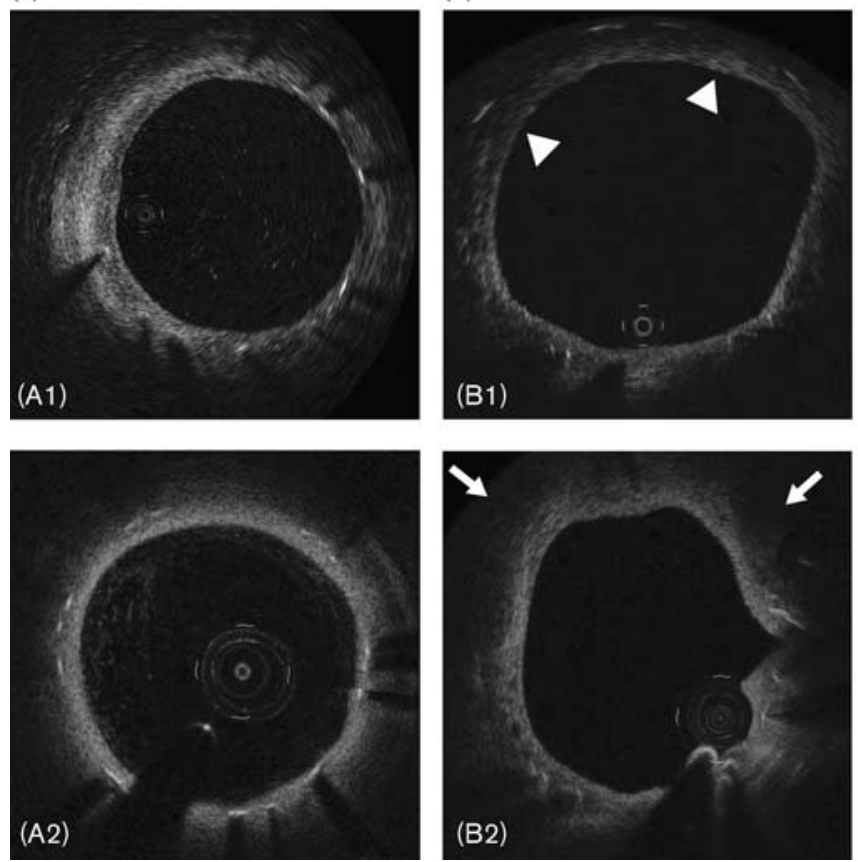

(c)
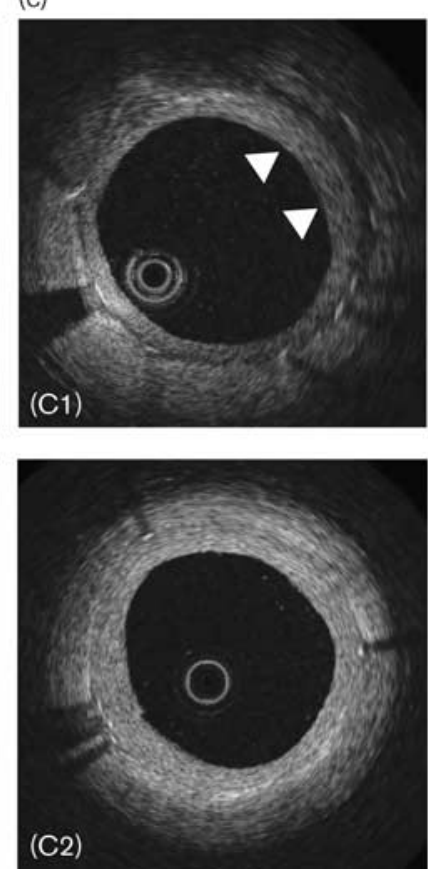

(d)
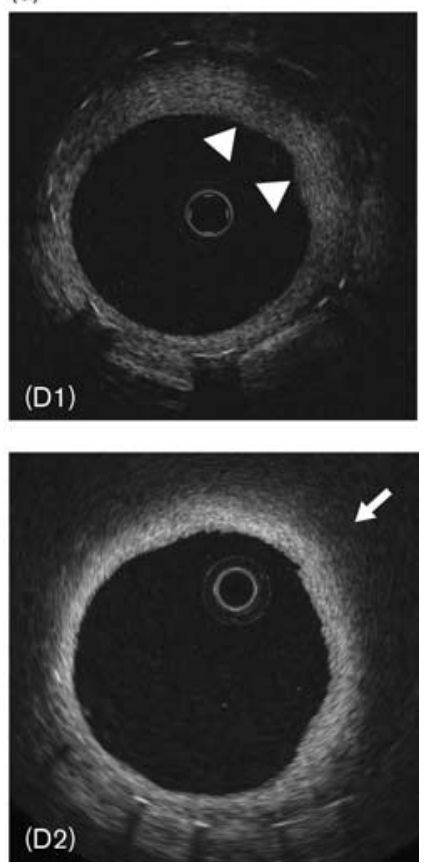

Representative images of the change of neointimal pattern. (A-1-D-1) Images at 6-month follow-up. (A-2-D-2) The corresponding images at the 12-month follow-up. (a) From a heterogeneous to a homogeneous pattern; (b) from a heterogeneous pattern (arrowhead) to a neoatherosclerosis pattern (arrow); (c) from a layered pattern (arrowhead) to a homogeneous pattern; and (d) from a layered pattern (arrowhead) to a neoatherosclerosis (arrow) pattern.

layered patterns correlated with more than one histological pattern [26]. The outcome of different patterns of neointima may be dictated by the predominant component within the neointima. For example, fibrin, which is observed more frequently in DES than in a bare-metal stent (BMS) [27], generally disappears during follow-up [28]. However, proteoglycan deposition, which promotes retention of lipoprotein, and infiltration of inflammatory cells such as macrophages, lymphocytes, and giant cells contribute toward the development of NA [29,30]. These cells may gradually replace smooth muscle cells, resulting in changes in the neointimal pattern and eventually the development of NA. In the current study, progression from a layered to a heterogeneous pattern was not detected. A recent pathology study reported that $59 \%$ of frames with a layered pattern corresponded to healed rupture/erosion and $6 \%$ involved neointimal calcification. However, $86 \%$ of frames with a heterogeneous pattern showed hypersensitivity vasculitis and $14 \%$ showed smooth muscle cells in a proteoglycan-rich and collagen-rich tissue [26]. These findings may suggest that layered pattern neointima might be more mature than heterogeneous patterns. Therefore, changes from a layered pattern to a heterogeneous pattern may not occur.

\section{Progression to neoatherosclerosis}

Progression to NA was observed in $0.6 \%$ of patients with a homogeneous pattern, $5.6 \%$ of patients with a heterogeneous pattern, and $3.9 \%$ of patients with a layered pattern at the 6-month follow-up. Previous studies reported a higher incidence of NA compared with our study. However, the follow-up duration of these studies was longer than ours. This indicates that neoatherosclerotic changes that can be detected by OCT mainly occur after 1 year. Although this could not be studied because of small numbers of cases, different neointimal patterns may reflect different underlying mechanisms of development of NA. NA in BMS is typically manifested as a lipid pool in a well-developed neointima, which is rich in vascular smooth muscle cells, and apoptosis of vascular smooth muscle cells is believed to be the underlying cause of NA [31]. However, NA in DES is considered to be a direct consequence of delayed artery healing with macrophage infiltration and apoptosis. Indeed, NA with restenosis was found in no more than $20 \%$ of the entire NA population with DES [3]. In addition, our group previously reported the association between the degree of neointima hyperplasia and the incidence of NA [32]. The incidence of NA was higher in DES than in BMS in the early phase, despite thinner neointima. In addition, heterogeneous neointima had thinner neointima than layered pattern neointima (mean neointimal thickness 347.1 vs. $533.9 \mu \mathrm{m}, P<0.001)$. As mentioned above, fibrin deposition, which suggests delayed arterial healing in DES, was found in heterogeneous pattern neointima. Indeed, the presence of fibrin was more frequently observed and the degree of 
neointima hyperplasia was significantly smaller in heterogeneous than in layered patterns in the validation study in the swine model [33]. Heterogeneous neointima that has relatively thin neointima might be closely associated with the process leading to NA in DES.

\section{Regression to a homogeneous pattern}

Conversion to a homogeneous pattern was observed in $5.6 \%$ of patients with heterogeneous neointima and of $11.5 \%$ of patients with layered pattern neointima. Homogeneous pattern neointima is mainly composed of fibrous connective tissue and the conversion to a homogeneous pattern was considered to be the normal healing process, and associated with a more favorable outcome $[33,34]$. In addition, the prevalence of PLIA also decreased from the 6-month follow-up to the 12-month follow-up. PLIA also include dark appearance areas in OCT, and the corresponding histologic tissues are reported to be fibrin and the myxomatous extracellular matrix including proteoglycan [20]. There are no objective criteria for the diagnosis of PLIA. Therefore, PLIA might also partly overlap with heterogeneous and layered patterns. As discussed above, dark appearance areas on OCT may represent various tissues, and some of those tissues may later be replaced by collagen fiber. Therefore, the reversibility of those dark appearance areas on OCT in early phases is not surprising. Because of the number of cases, we could not study the factors associated with regression or progression of neointima. A previous study reported that NA is more common in patients with native atherosclerosis progression [35]. Some other studies reported that serum low-density lipoprotein levels and the presence of chronic kidney disease are independent predictors for NA $[6,9,10]$. One study reported that optimal lipid-lowing therapy prevented conversion from homogeneous to nonhomogeneous pattern intima [36]. These results suggest that not only focal vascular response but also systemic factors might influence the changes in the neointimal pattern. Further study is warranted to determine the factors for predicting the changes in the neointimal pattern and for understanding the association between those changes in the neointimal pattern and clinical outcomes.

\section{Limitations}

There are several limitations in this study. First, this is a retrospective observational study. Second, stents with severe in-stent restenosis at the 6-month follow-up were excluded because new stents were implanted in these cases and a 12-month follow-up study could not be performed. Third, the follow-up period was short to detect the serial change in NA. It is difficult to carry out a followup OCT study after several years in completely asymptomatic patients. Therefore, we used the data set that included patients who underwent scheduled follow-up at 12 months. Fourth, the number of second-generation DES in this study was small. Therefore, we could not compare the different neointimal patterns between the different DES types.

\section{Conclusion}

Most patients had homogenous pattern neointima both at 6 and at 12 months. In those with neointima thickness greater than $100 \mu \mathrm{m}$ at 6 months, $8.0 \%$ of homogenous neointima progressed to a nonhomogeneous pattern and $9.1 \%$ of nonhomogeneous neointima converted into a homogeneous pattern at 12 months. Further studies are needed to understand the mechanisms of these neointimal changes and their clinical significance.

\section{Acknowledgements}

Dr. Jang's research was supported by 'Mr. and Mrs. Michael and Kathryn Park' and by 'Mrs. and Mr. Gill and Allan Gray'. Aaron Aguirre is supported by a grant from the American Heart Association (14FTF20380185).

\section{Conflicts of interest}

Dr. Jang has received grants and consulting fees from St. Jude Medical/Abbott. For the remaining authors there are no conflicts of interest.

\section{References}

1 Park S-J, Kang S-J, Virmani R, Nakano M, Ueda Y. In-stent neoatherosclerosis: a final common pathway of late stent failure. $J \mathrm{Am}$ Coll Cardiol 2012; 59:2051-2057.

2 Nakazawa G, Otsuka F, Nakano M, Vorpahl M, Yazdani SK, Ladich E, et al. The pathology of neoatherosclerosis in human coronary implants bare-metal and drug-eluting stents. J Am Coll Cardiol 2011; 57:1314-1322.

3 Otsuka F, Byrne RA, Yahagi K, Mori H, Ladich E, Fowler DR, et al. Neoatherosclerosis: overview of histopathologic findings and implications for intravascular imaging assessment. Eur Heart J 2015; 36:2147-2159.

4 Taniwaki M, Radu MD, Zaugg S, Amabile N, Garcia-Garcia HM, Yamaji K, et al. Mechanisms of very late drug-eluting stent thrombosis assessed by optical coherence tomography. Circulation 2016; 133:650-660.

5 Otsuka F, Vorpahl M, Nakano M, Foerst J, Newell JB, Sakakura K, et al. Pathology of second-generation everolimus-eluting stents versus firstgeneration sirolimus- and paclitaxel-eluting stents in humans. Circulation 2014; 129:211-223.

6 Lee S-Y, Hur S-H, Lee S-G, Kim S-W, Shin D-H, Kim J-S, et al. Optical coherence tomographic observation of in-stent neoatherosclerosis in lesions with more than $50 \%$ neointimal area stenosis after second-generation drug-eluting stent implantation. Circ Cardiovasc Interv 2015; 8:e001878-e001878.

7 Bastante T, Rivero F, Benedicto A, Cuesta J, Alfonso F. Recurrent neoatherosclerosis after bioresorbable vascular scaffold treatment of in-stent restenosis. JACC Cardiovasc Interv 2015; 8:1264-1265.

8 Sato T, Richardt G, Abdel-Wahab M. Early neoatherosclerosis after bioresorbable vascular scaffold implantation: insights from optical coherence tomography. Coron Artery Dis 2016; 27:616-617.

9 Yonetsu T, Kato K, Kim S-J, Xing L, Jia H, McNulty I, et al. Predictors for neoatherosclerosis: a retrospective observational study from the optical coherence tomography registry. Circ Cardiovasc Imaging 2012; 5:660-666.

10 Kuroda M, Otake H, Shinke T, Takaya T, Nakagawa M, Osue T, et al. The impact of in-stent neoatherosclerosis on long-term clinical outcomes: an observational study from the Kobe University Hospital optical coherence tomography registry. Eurolntervention 2015; 12:e1366-e1374.

11 Lee SY, Hong MK, Mintz GS, Shin DH, Kim JS, Kim BK, et al. Temporal course of neointimal hyperplasia following drug-eluting stent implantation: a serial follow-up optical coherence tomography analysis. Int J Cardiovasc Imaging 2014; 30:1003-1011.

12 Kim JS, Hong MK, Shin DH, Kim BK, Ko YG, Choi D, et al. Quantitative and qualitative changes in DES-related neointimal tissue based on serial OCT. JACC Cardiovasc Imaging 2012; 5:1147-1155.

13 Kato K, Yonetsu T, Kim SJ, Xing L, Lee H, McNulty l, et al. Nonculprit plaques in patients with acute coronary syndromes have more vulnerable features 
compared with those with non-acute coronary syndromes a 3-vessel optical coherence tomography study. Circ Cardiovasc Imaging 2012; 5:433-440.

14 Kato K, Yonetsu T, Kim S-J, Xing L, Lee H, McNulty I, et al. Comparison of nonculprit coronary plaque characteristics between patients with and without diabetes: a 3-vessel optical coherence tomography study. JACC Cardiovasc Interv 2012; 5:1150-1158.

15 Gonzalo N, Serruys PW, Okamura T, van Beusekom HM, Garcia-Garcia HM, van Soest $\mathrm{G}$, et al. Optical coherence tomography patterns of stent restenosis. Am Heart J 2009; 158:284-293.

16 Takano M, Yamamoto M, Inami S, Murakami D, Ohba T, Seino Y, et al. Appearance of lipid-laden intima and neovascularization after implantation of bare-metal stents. Extended late-phase observation by intracoronary optical coherence tomography. J Am Coll Cardiol 2009; 55:26-32.

17 Imanaka T, Fujii K, Hao H, Shibuya M, Saita T, Kawakami R, et al. Ex vivo assessment of neointimal characteristics after drug-eluting stent implantation: optical coherence tomography and histopathology validation study. Int J Cardiol 2016; 221:1043-1047.

18 Di Vito L, Yoon JH, Kato K, Yonetsu T, Vergallo R, Costa M, et al. Comprehensive overview of definitions for optical coherence tomography based plaque and stent analyses. Coron Artery Dis 2014; 25:172-185.

19 Otake H, Shite J, Ikeno F, Shinke T, Teramoto T, Miyoshi N, et al. Evaluation of the peri-strut low intensity area following sirolimus- and paclitaxel-eluting stents implantation: Insights from an optical coherence tomography study in humans. Int J Cardiol 2012; 157:38-42.

20 Teramoto T, Ikeno F, Otake H, Lyons JK, van Beusekom HMM, Fearon WF, et al. Intriguing peri-strut low-intensity area detected by optical coherence tomography after coronary stent deployment. Circ J 2010; 74:1257-1259.

21 Tearney GJ, Yabushita H, Houser SL, Aretz HT, Jang IK, Schlendorf KH, et al. Quantification of macrophage content in atherosclerotic plaques by optical coherence tomography. Circulation 2003; 107:113-119.

22 MacNeill BD, Jang IK, Bouma BE, Iftimia N, Takano M, Yabushita H, et al. Focal and multi-focal plaque macrophage distributions in patients with acute and stable presentations of coronary artery disease. J Am Coll Cardio/ 2004; 44:972-979.

23 Habara M, Terashima M, Nasu K, Kaneda H, Yokota D, Ito T, et al. Morphological differences of tissue characteristics between early, late, and very late restenosis lesions after first generation drug-eluting stent implantation: an optical coherence tomography study. Eur Hear $J$ Cardiovasc Imaging 2013; 14:276-284.

24 Nakano M, Vorpahl M, Otsuka F, Taniwaki M, Yazdani SK, Finn AV, et al. Ex vivo assessment of vascular response to coronary stents by optical frequency domain imaging. JACC Cardiovasc imaging 2012; 5:71-82.
25 Shibuya M, Fujii K, Hao H, Imanaka T, Saita T, Fukunaga M, et al. Tissue Characterization of in-stent neointima using optical coherence tomography in the late phase after bare-metal stent implantation - an ex vivo validation study. Circ J 2015; 79:2224-2230.

26 Lutter C, Mori H, Yahagi K, Ladich E, Joner M, Kutys R, et al. Histopathological differential diagnosis of optical coherence tomographic image interpretation after stenting. JACC Cardiovasc Interv 2016; 9:2511-2523.

27 Joner M, Finn AV, Farb A, Mont EK, Kolodgie FD, Ladich E, et al. Pathology of drug-eluting stents in humans: delayed healing and late thrombotic risk. J Am Coll Cardiol 2006; 48:193-202.

28 Nakazawa G, Finn AV, John MC, Kolodgie FD, Virmani R. The significance of preclinical evaluation of sirolimus-, paclitaxel-, and zotarolimus-eluting stents. Am J Cardiol 2007; 100:36M-44M.

29 Nakano M, Otsuka F, Yahagi K, Sakakura K, Kutys R, Ladich ER, et al. Human autopsy study of drug-eluting stents restenosis: histomorphological predictors and neointimal characteristics. Eur Heart J 2013; 34:3304-3313.

30 Nakazawa G, Ladich E, Finn AV, Virmani R. Pathophysiology of vascular healing and stent mediated arterial injury. Eurolntervention 2008; 4 (Suppl C):7-10.

31 Romero ME, Yahagi K, Kolodgie FD, Virmani R. Neoatherosclerosis from a pathologist's point of view. Arterioscler Thromb Vasc Biol 2015; 35: e43-e49.

32 Vergallo R, Yonetsu T, Uemura S, Park S-J, Lee S, Kato K, et al. Correlation between degree of neointimal hyperplasia and incidence and characteristics of neoatherosclerosis as assessed by optical coherence tomography. Am J Cardiol 2013; 112:1315-1321.

33 Kim J-S, Afari ME, Ha J, Tellez A, Milewski K, Conditt G, et al. Neointimal patterns obtained by optical coherence tomography correlate with specific histological components and neointimal proliferation in a swine model of restenosis. Eur Hear J Cardiovasc Imaging 2014; 15:292-298.

34 Yabushita $\mathrm{H}$. Characterization of human atherosclerosis by optical coherence tomography. Circulation 2002; 106:1640-1645.

35 Taniwaki M, Windecker S, Zaugg S, Stefanini GG, Baumgartner S, Zanchin $\mathrm{T}$, et al. The association between in-stent neoatherosclerosis and native coronary artery disease progression: a long-term angiographic and optical coherence tomography cohort study. Eur Heart J 2015; 36:2167-2176.

36 Jang JY, Kim JS, Shin DH, Kim BK, Ko YG, Choi D, et al. Favorable effect of optimal lipid-lowering therapy on neointimal tissue characteristics after drugeluting stent implantation: Qualitative optical coherence tomographic analysis. Atherosclerosis 2015; 242:553-559. 